\title{
K-BESSEL FUNCTIONS ASSOCIATED TO A 3-RANK JORDAN ALGEBRA
}

\author{
HACEN DIB
}

Received 2 January 2005 and in revised form 8 September 2005

Using the Bessel-Muirhead system, we can express the $K$-Bessel function defined on a Jordan algebra as a linear combination of the J-solutions. We determine explicitly the coefficients when the rank of this Jordan algebra is three after a reduction to the rank two. The main tools are some algebraic identities developed for this occasion.

\section{Introduction}

Bessel functions of matrix argument appeared as a subject of studies in the work of Herz [6]. One can find in the multivariate statistics literature some applications of these functions (see [11]). The main tool of Herz's work was the Laplace transform and its inverse in the space of real symmetric matrices. He obtained several properties but a "good" differential system was lacking. This was the major contribution of Muirhead in [10] when he characterized them by a system of second-order partial differential equations and proved the uniqueness (up to multiplicative constant) of the solution which is analytic at 0 . Later, Faraut and Travaglini [5] gave a generalization of these functions to a Jordan algebra. An extensive study was done in [2]. However, the explicit resolution of the Bessel-Muirhead system in general rank remains an open problem. Nevertheless, in [9] Mahmoud wrote down an explicit basis of the solutions in the rank 2 and 3 using series of one-variable Bessel functions. On the other hand, the $K$-Bessel function of matrix argument was defined earlier in Herz's paper cited above and his conjecture was that there must be a linear relation between this kind of Bessel functions and the J's one as known in the onevariable theory. The first result concerning this conjecture was established by the author in [3] for the rank two. In this paper, we continue our work and prove that a similar result for the $K$-Bessel function is also true when the Jordan algebra is of rank 3 . In this case, there are four nonequivalent classes of real simple and Euclidean Jordan algebra: $\operatorname{Herm}(3, \mathbb{F})$ the algebra of $3 \times 3$ Hermitian matrices, where $\mathbb{F}$ is the field of real, complex, quaternionic, or Cayley (octaves) numbers. In [3] we intended to perform a case-by-case calculation. In this way, a serious difficulty arises in the evaluation of some integral over the automorphism group of the Jordan algebra. However, a unified treatment is possible 
$2864 K$-Bessel functions associated to a 3-rank Jordan algebra

by a reduction to the rank-2 case using some algebraic identities. This is what we will present here.

\section{Preliminaries}

Let us recall the situation and some useful results in [3].

Definition 2.1. Bessel-Muirhead operators are defined by

$$
B_{i}=x_{i} \frac{\partial^{2}}{\partial x_{i}^{2}}+(\nu+1) \frac{\partial}{\partial x_{i}}+1+\frac{d}{2} \sum_{j \neq i} \frac{1}{x_{i}-x_{j}}\left(x_{i} \frac{\partial}{\partial x_{i}}-x_{j} \frac{\partial}{\partial x_{j}}\right), \quad 1 \leq i \leq r,
$$

where $r$ is the rank of the system, $v$ a complex parameter, and $d$ an integer (which will be interpreted below in Section 3). A Bessel function is a symmetric function $f$ which is a solution of the Bessel-Muirhead system $B_{i} f=0, i=1,2, \ldots, r$.

Denote by $t_{1}, t_{2}, \ldots, t_{r}$ the elementary symmetric functions, that is,

$$
t_{p}=\sum_{1 \leq i_{1}<i_{2}<\cdots<i_{p} \leq r} x_{i_{1}} x_{i_{2}} \cdots x_{i_{p}}
$$

with $t_{0}=1$ and $t_{p}=0$ if $p<0$ or $p>r$. The Bessel-Muirhead system is then equivalent to the system (see $[2,7]) Z_{k} g=0,1 \leq k \leq r$, where

$$
\begin{gathered}
Z_{k}=\sum_{i, j=1}^{r} A_{i j}^{k} \frac{\partial^{2}}{\partial t_{i} \partial t_{j}}+\left(\nu+1+\frac{r-k}{2} d\right) \frac{\partial}{\partial t_{k}}+\delta_{k}^{1}, \\
A_{i j}^{k}= \begin{cases}t_{i+j-k} & \text { if } i, j \geq k, \\
-t_{i+j-k} & \text { if } i, j<k, i+j \geq k, \\
0 & \text { elsewhere. }\end{cases}
\end{gathered}
$$

Here $\delta_{k}^{1}$ is the Kronecker symbol and $g\left(t_{1}, t_{2}, \ldots, t_{r}\right)=f\left(x_{1}, x_{2}, \ldots, x_{r}\right)$. When $r=2$, we have (see [3]) a fundamental set of solutions given by $J_{\nu}^{[2,1]}, J_{\nu}^{[2,2]}, t_{2}^{-\nu} J_{-\nu}^{[2,1]}$, and $t_{2}^{-\nu} J_{-\nu}^{[2,2]}$, where

$$
\begin{gathered}
J_{\nu}^{[2,1]}\left(t_{1}, t_{2}\right)=\sum_{m_{1}, m_{2} \geq 0} \frac{(-1)^{m_{1}}}{(1)_{m_{1}}(1)_{m_{2}}(1+v)_{m_{2}}(1+v+d / 2)_{m_{1}+2 m_{2}}} t_{1}^{m_{1}} t_{2}^{m_{2}}, \\
J_{\nu}^{[2,2]}\left(t_{1}, t_{2}\right)=\sum_{m_{1}, m_{2} \geq 0} \frac{(-1)^{m_{1}}}{(1-v-d / 2)_{m_{1}}(1)_{m_{2}}(1+v)_{m_{2}}(1)_{m_{1}+2 m_{2}}} t_{1}^{m_{1}-v-d / 2} t_{2}^{m_{2}} .
\end{gathered}
$$

Here $(a)_{k}$ is the classical Pochhammer symbol, that is, $(a)_{k}=a(a+1) \cdots(a+k-1)$. In this case, the $K$-Bessel function (in two variables) can be expressed (in the J-basis) as follows (see [3]):

$$
K_{\nu}^{[2]}\left(t_{1}, t_{2}\right)=\sum_{j=1}^{2} a_{\nu}^{j} J_{\nu}^{[2, j]}\left(-t_{1}, t_{2}\right)+b_{\nu}^{j} t_{2}^{-v} J_{-v}^{[2, j]}\left(-t_{1}, t_{2}\right),
$$


where

$$
\begin{aligned}
& a_{v}^{1}=b_{-v}^{1}=(2 \pi)^{(n-2) / 2} \Gamma(-v) \Gamma\left(-v-\frac{n-2}{2}\right), \\
& a_{v}^{2}=b_{-v}^{2}=(2 \pi)^{(n-2) / 2} \Gamma(-v) \Gamma\left(\nu+\frac{n-2}{2}\right)
\end{aligned}
$$

(for the meaning of $n$, see Section 3 below). Observe that this expression reduces (up to constant factor) to the classical one-variable formula when $t_{2}=0$ and generic $v$ (i.e., $v \notin Z$ and $\nu \pm(n-2) / 2 \notin Z)$. Now, for $r=3$, we have eight linearly independent J-solutions $J_{\nu}^{[3,1]}, J_{\nu}^{[3,2]}, J_{\nu}^{[3,3]}, J_{\nu}^{[3,4]}, t_{3}^{-v} J_{-\nu}^{[3,1]}, t_{3}^{-v} J_{-\nu}^{[3,2]}, t_{3}^{-v} J_{-\nu}^{[3,3]}$, and $t_{3}^{-v} J_{-\nu}^{[3,4]}$, where

$$
\begin{aligned}
& J_{\nu}^{[3,1]}\left(t_{1}, t_{2}, t_{3}\right)=\sum_{m_{1}, m_{2}, m_{3} \geq 0} \frac{(-1)^{m_{1}+m_{3}}}{(1)_{m_{1}}(1)_{m_{2}}(1)_{m_{3}}(1+\nu)_{m_{3}}(1+v+d / 2)_{m_{2}+2 m_{3}}} \\
& \times \frac{(1+2 v+d)_{m_{1}+2 m_{2}+4 m_{3}}}{(1+v+d)_{m_{1}+2 m_{2}+3 m_{3}}(1+2 v+d)_{m_{1}+2 m_{2}+3 m_{3}}} t_{1}^{m_{1}} t_{2}^{m_{2}} t_{3}^{m_{3}}, \\
& J_{\nu}^{[3,2]}\left(t_{1}, t_{2}, t_{3}\right)=\sum_{m_{1}, m_{2}, m_{3} \geq 0} \frac{(-1)^{m_{1}+m_{3}}}{(1-v-d)_{m_{1}}(1)_{m_{2}}(1)_{m_{3}}(1+\nu)_{m_{3}}(1+v+d / 2)_{m_{2}+2 m_{3}}} \\
& \times \frac{(1+\nu)_{m_{1}+2 m_{2}+4 m_{3}}}{(1)_{m_{1}+2 m_{2}+3 m_{3}}(1+v)_{m_{1}+2 m_{2}+3 m_{3}}} t_{1}^{m_{1}-v-d} t_{2}^{m_{2}} t_{3}^{m_{3}}, \\
& J_{\nu}^{[3,3]}\left(t_{1}, t_{2}, t_{3}\right)=\sum_{m_{1}, m_{2}, m_{3} \geq 0} \frac{(-1)^{m_{1}+m_{3}}}{(1)_{m_{1}}(1-v-d / 2)_{m_{2}}(1)_{m_{3}}(1+\nu)_{m_{3}}(1)_{m_{2}+2 m_{3}}} \\
& \times \frac{(1)_{m_{1}+2 m_{2}+4 m_{3}}}{(1-v)_{m_{1}+2 m_{2}+3 m_{3}}(1)_{m_{1}+2 m_{2}+3 m_{3}}} t_{1}^{m_{1}} t_{2}^{m_{2}-\nu-d / 2} t_{3}^{m_{3}}, \\
& J_{\nu}^{[3,4]}\left(t_{1}, t_{2}, t_{3}\right)=\sum_{m_{1}, m_{2}, m_{3} \geq 0} \frac{(-1)^{m_{1}+m_{3}}}{(1+\nu)_{m_{1}}(1-v-d / 2)_{m_{2}}(1)_{m_{3}}(1+v)_{m_{3}}(1)_{m_{2}+2 m_{3}}} \\
& \times \frac{(1+\nu)_{m_{1}+2 m_{2}+4 m_{3}}}{(1)_{m_{1}+2 m_{2}+3 m_{3}}(1+\nu)_{m_{1}+2 m_{2}+3 m_{3}}} t_{1}^{m_{1}+v} t_{2}^{m_{2}-\nu-d / 2} t_{3}^{m_{3}} .
\end{aligned}
$$

Observe also that when $t_{3}=0$ (and $\nu \notin Z, v \pm d / 2 \notin Z$ ), these functions reduce to

$$
\begin{aligned}
& J_{\nu}^{[3,1]}\left(t_{1}, t_{2}, 0\right)=J_{\nu+d / 2}^{[2,1]}\left(t_{1}, t_{2}\right), \\
& J_{\nu}^{[3,2]}\left(t_{1}, t_{2}, 0\right)=J_{\nu+d / 2}^{[2,2]}\left(t_{1}, t_{2}\right), \\
& J_{\nu}^{[3,3]}\left(t_{1}, t_{2}, 0\right)=t_{2}^{-v-d / 2} J_{-\nu-d / 2}^{[2,1]}\left(t_{1}, t_{2}\right), \\
& J_{\nu}^{[3,4]}\left(t_{1}, t_{2}, 0\right)=t_{2}^{-v-d / 2} J_{-\nu-d / 2}^{[2,2]}\left(t_{1}, t_{2}\right) .
\end{aligned}
$$

This behaviour and the expression of $K_{\nu}^{[2]}$ are an important step to the final result.

\section{Some algebraic identities}

For the general theory of Jordan algebra, one can see [4], but what we will develop is somehow specific to the rank three. So, let $A$ be a real simple and Euclidean Jordan 
algebra with rank 3 and real dimension $n$. We know that $n=3+3 d$, where $d=1,2,4$, or 8 . The integer $d$ is the real dimension of some decomposition spaces which appear in the Peirce decomoposition of the algebra $A$ (see [4]). Let $\left\{c_{1}, c_{2}, c_{3}\right\}$ be a complete system of mutually orthogonal primitive idempotents, that is, $c_{i} c_{j}=\delta_{i}^{j} c_{i}, c_{1}+c_{2}+c_{3}=e$ the unit of $A$ and none of the $c_{j}$ 's can split into a sum of two idempotents. We have a CayleyHamilton-like theorem $x^{3}-a_{1}(x) x^{2}+a_{2}(x) x-a_{3}(x) e=0$ and a spectral decomposition $x=k \cdot\left(\lambda_{1} c_{1}+\lambda_{2} c_{2}+\lambda_{3} c_{3}\right)$ with $k$ an element of the automorphism group of $A$ and $\lambda_{i}$ reals such that

$$
\begin{aligned}
& a_{1}(x)=\lambda_{1}+\lambda_{2}+\lambda_{3}:=\operatorname{tr}(x), \\
& a_{3}(x)=\lambda_{1} \lambda_{2} \lambda_{3}:=\operatorname{det}(x), \\
& a_{2}(x)=\lambda_{1} \lambda_{2}+\lambda_{1} \lambda_{3}+\lambda_{2} \lambda_{3}=\frac{1}{2}\left[\operatorname{tr}(x)^{2}-\operatorname{tr}\left(x^{2}\right)\right] .
\end{aligned}
$$

The inner product is defined then by $(x, y):=\operatorname{tr}(x y)$. The operators $L(x)$ and $P(x)$ are defined by $L(x) y=x y$ and $P(x)=2 L^{2}(x)-L\left(x^{2}\right)$. Let us consider the Peirce decomposition with respect to the idempotent $c_{3}$, that is, $A=A_{0} \oplus A_{1 / 2} \oplus A_{1}$, where $A_{\alpha}$ is the eigenspace of $L\left(c_{3}\right)$ with respect to the eigenvalue $\alpha . A_{0}$ and $A_{1}$ are Jordan subalgebras of rank 2 and 1, respectively, and $A_{1 / 2}$ is a subspace of dimension $2 d$. Put $n_{0}=\operatorname{dim} A_{0}=2+d$ and $e_{0}=c_{1}+c_{2}$ the unit of $A_{0}$. We have $A_{0} A_{1}=\{0\},\left(A_{0} \oplus A_{1}\right) A_{1 / 2} \subset A_{1 / 2}$ and $A_{1 / 2} A_{1 / 2} \subset$ $A_{0} \oplus A_{1}$. If we write $\operatorname{tr}(z)$ or $\operatorname{det}(z)$ of an element of $A_{0}$, this will mean trace and determinant with respect to the subalgebra $A_{0}$. We denote by $\Omega_{3}$ the cone of positivity of $A$, that is, $\Omega_{3}=\left\{x \in A / \lambda_{i}>0, i=1,2,3\right\}=\left\{x \in A / a_{i}(x)>0, i=1,2,3\right\}$ and by $\Omega_{2}$ the cone of $A_{0}$. Every $x$ in $\Omega_{3}$ (resp., in $\Omega_{2}$ ) admits a unique square root in $\Omega_{3}$ (resp., in $\Omega_{2}$ ) and is invertible.

Lemma 3.1. If $y=e_{0}+\xi+t c_{3}$ with $\xi \in A_{1 / 2}$ and $t \in \mathbb{R}$, then

$$
\operatorname{det}(y)=t-\frac{1}{2}\|\xi\|^{2}
$$

Proof. The projection onto $A_{1}$ is $P\left(c_{3}\right)$, so $P\left(c_{3}\right) \xi=0$ and therefore $0=\operatorname{tr}\left(P\left(c_{3}\right) \xi\right)=$ $\left(e, P\left(c_{3}\right) \xi\right)=\left(c_{3}, \xi\right)=(1 / 2) \operatorname{tr}(\xi)$. By the same argument $\operatorname{tr}\left(\xi^{3}\right)=0$. Now by CayleyHamilton $\xi^{3}+a_{2}(\xi) \xi-\operatorname{det}(\xi) e=0$ which implies $\operatorname{det}(\xi)=0$. On the other hand, $\xi^{2}=$ $u+\tau c_{3}$ with $u \in A_{0}$ and $\tau \in \mathbb{R}$. We have $\tau=\left(c_{3}, \xi^{2}\right)=\left(\xi c_{3}, \xi\right)=(1 / 2)\|\xi\|^{2}$ and $\operatorname{tr}(u)=$ $(1 / 2)\|\xi\|^{2}$. From $\operatorname{det}\left(\xi^{2}\right)=\tau \operatorname{det}(u)$, we deduce that $\operatorname{det}(u)=0$. So by Cayley-Hamilton (in $A_{0}$ ) we can write $u^{2}=(1 / 2)\|\xi\|^{2} u$. Now $\xi^{2}=u+\tau c_{3} \Rightarrow \xi^{4}=u^{2}+\tau^{2} c_{3}=(1 / 2)\|\xi\|^{2} u+$ $(1 / 4)\|\xi\|^{4} c_{3}=(1 / 2)\|\xi\|^{2} \xi^{2}$ and then $\xi^{3}=(1 / 2)\|\xi\|^{2} \xi$. Therefore, $u \xi=(1 / 4)\|\xi\|^{2} \xi$. Then we have

$$
\begin{aligned}
y & =e_{0}+\xi+t c_{3} \\
y^{2} & =\left(e_{0}+u\right)+(1+t) \xi+\left(t^{2}+\frac{1}{2}\|\xi\|^{2}\right) c_{3}, \\
y^{3} & =\left(e_{0}+(2+t) u\right)+\left(1+t+t^{2}+\frac{1}{2}\|\xi\|^{2}\right) \xi+\left(t^{2}+t\|\xi\|^{2}+\frac{1}{2}\|\xi\|^{2}\right) c_{3},
\end{aligned}
$$


and also $\operatorname{tr}(y)=2+t, a_{2}(y)=(1 / 2)\left[\operatorname{tr}(y)^{2}-\operatorname{tr}\left(y^{2}\right)\right]=1+2 t-(1 / 2)\|\xi\|^{2}$. The result is a consequence of

$$
y^{3}-\operatorname{tr}(y) y^{2}+a_{2}(y) y=\left(t-\frac{1}{2}\|\xi\|^{2}\right) e .
$$

LemmA 3.2. If $y=z+\xi+t c_{3}$ with $z \in \Omega_{2}, \xi \in A_{1 / 2}$, and $t \in \mathbb{R}$, then

$$
\operatorname{det}(y)=\operatorname{det}(z)\left[t-\left(z^{-1} \xi, \xi\right)\right]
$$

Proof. The application

$$
\begin{gathered}
\rho: A_{0} \longrightarrow \text { End }\left(A_{1 / 2}\right), \\
u \longrightarrow \rho(u) \text { defined by } \rho(u) \xi=2 L(u) \xi
\end{gathered}
$$

is a representation of $A_{0}$ in the space $A_{1 / 2}$ (for more details, see [4]). This means that $2 \rho(u v)=\rho(u) \rho(v)+\rho(v) \rho(u)$ and identically $P(u) \xi=0$. So

$$
\begin{aligned}
P\left(u+c_{3}\right) y & =P\left(u+c_{3}\right) z+P\left(u+c_{3}\right) \xi+t P\left(u+c_{3}\right) c_{3} \\
& =P(u) z+2 L(u) \xi+t c_{3} .
\end{aligned}
$$

Now if $u=z^{-1 / 2}$, we derive the desired result thanks to (3.2) and the fact that

$$
\begin{aligned}
\operatorname{det}\left[P\left(z^{-1 / 2}+c_{3}\right) y\right] & =\operatorname{det}\left(z^{-1 / 2}+c_{3}\right)^{2} \operatorname{det}(y) \\
& =\operatorname{det}\left(z^{-1}\right) \operatorname{det}(y)=\operatorname{det}(z)^{-1} \operatorname{det}(y) .
\end{aligned}
$$

Corollary 3.3. If $y=z+\xi+t c_{3} \in \Omega_{3}$, then

$$
\operatorname{tr}\left(y^{-1}\right)=\frac{2 \operatorname{det}(z)+2 t \operatorname{tr}(z)-\|\xi\|^{2}}{2 \operatorname{det}(z)\left[t-\left(z^{-1} \xi, \xi\right)\right]} .
$$

Proof. First we have $y^{3}-\operatorname{tr}(y) y^{2}+a_{2}(y) y-\operatorname{det}(y) e=0$. Then

$$
y^{-1}=\frac{1}{\operatorname{det}(y)}\left[y^{2}-\operatorname{tr}(y) y+a_{2}(y) e\right]
$$

and therefore

$$
\begin{aligned}
\operatorname{tr}\left(y^{-1}\right) & =\frac{1}{\operatorname{det}(y)}\left[\operatorname{tr}\left(y^{2}\right)-\operatorname{tr}(y)^{2}+3 a_{2}(y)\right] \\
& =\frac{1}{2 \operatorname{det}(y)}\left[\operatorname{tr}(y)^{2}-\operatorname{tr}\left(y^{2}\right)\right] \\
& =\frac{1}{2 \operatorname{det}(y)}\left[\operatorname{tr}(z)^{2}+2 t \operatorname{tr}(z)-\operatorname{tr}\left(z^{2}\right)-\|\xi\|^{2}\right] \\
& =\frac{1}{2 \operatorname{det}(y)}\left[2 \operatorname{det}(z)+2 t \operatorname{tr}(z)-\|\xi\|^{2}\right] .
\end{aligned}
$$


$2868 K$-Bessel functions associated to a 3-rank Jordan algebra

\section{4. $K$-Bessel function}

The $K$-Bessel function is defined by (see $[2,4]$ )

$$
K_{\nu}^{[3]}(x)=\int_{\Omega_{3}} e^{-\operatorname{tr}\left(y^{-1}\right)-(x, y)}(\operatorname{det} y)^{\nu-n / 3} d y .
$$

This function is well defined at least for $x \in \Omega_{3}$ and any complex $\nu$. The convergence of the previous integral is assured by the good behaviour of $e^{-\operatorname{tr}\left(y^{-1}\right)-(x, y)}$ near infinity (in $\Omega_{3}$ ) and $\partial \Omega_{3}$. We can extend it to the tube $\Omega_{3} \oplus i A$ in the complexification $A_{\mathbb{C}}=A \oplus i A$ of $A$ as a holomorphic function in $x$. As a function of $v$, it is an entire function (see [4, Proposition XVI.3.1]). After a change of variables, one can show that

$$
K_{\nu}^{[3]}(x)=(\operatorname{det} x)^{-v} K_{-\nu}^{[3]}(x)
$$

Following [2] where it is proved that $K_{\nu}^{[3]}$ is a solution of a differential system similar to (2.1), we can write

$$
K_{\nu}^{[3]}(x)=\sum_{j=1}^{4} a_{\nu}^{j} J_{\nu}^{[3, j]}(-x)+b_{\nu}^{j} t_{3}^{-\nu} J_{-\nu}^{[3, j]}(-x)
$$

According to (4.2), we have $a_{v}^{j}=b_{-v}^{j}$ for $j=1,2,3,4$. For $\operatorname{Re}(\nu)<-d$, the following limit holds (see [4] for more information on $\Gamma_{\Omega_{3}}$, the Gamma function of the cone $\Omega_{3}$ ):

$$
\lim _{\substack{x \rightarrow 0 \\ x \in \Omega_{3}}} K_{\nu}^{[3]}(x)=\Gamma_{\Omega_{3}}(-\nu)=(2 \pi)^{3 d / 2} \Gamma(-\nu) \Gamma\left(-\nu-\frac{d}{2}\right) \Gamma(-\nu-d),
$$

so

$$
a_{v}^{1}=b_{-v}^{1}=(2 \pi)^{3 d / 2} \Gamma(-v) \Gamma\left(-v-\frac{d}{2}\right) \Gamma(-v-d)
$$

according to the behaviour of the solutions $J_{v}^{[3, j]}$. To determine the other coefficients, we take $x \neq 0$ on the boundary of $\Omega$. So if $x=x_{1} c_{1}+x_{2} c_{2}$, then the integral representation of $K_{\nu}^{[3]}$ takes the explicit form

$$
\begin{aligned}
K_{\nu}^{[3]}\left(x_{1} c_{1}+x_{2} c_{2}\right)=\int_{\Omega_{2}} \iint_{E} & \exp -\left[\frac{2 \operatorname{det}(z)+2 t \operatorname{tr}(z)-\|\xi\|^{2}}{2 \operatorname{det}(z)\left[t-\left(z^{-1} \xi, \xi\right)\right]}\right] \\
& \times \exp -(x, z) \operatorname{det}(z)^{\nu-d-1}\left[t-\left(z^{-1} \xi, \xi\right)\right]^{\nu-d-1} d z d \xi d t
\end{aligned}
$$

where $E=\left\{(t, \xi) \in \mathbb{R} \times A_{1 / 2} / t>\left(z^{-1} \xi, \xi\right)\right\}$ and $y=z+\xi+t c_{3}$. We change $t$ into $t+\left(z^{-1} \xi, \xi\right)$. The integral over $E$ becomes

$$
\begin{aligned}
I & =\int_{0}^{+\infty} \int_{A_{1 / 2}} e^{-1 / t} \exp -\left[\frac{\operatorname{tr}(z)\left(z^{-1} \xi, \xi\right)-(1 / 2)\|\xi\|^{2}}{t \operatorname{det}(z)}\right] t^{\nu-d-1} d \xi d t \\
& =\int_{0}^{+\infty} \int_{A_{1 / 2}} e^{-1 / t} e^{-(B \cdot \xi, \xi)} t^{\nu-d-1} d \xi d t
\end{aligned}
$$


where the operator $B=\rho(v)$ with $v=(\operatorname{tr}(z) / 2 t \operatorname{det}(z)) z^{-1}-(1 / 2 t \operatorname{det}(z)) e_{0}$. Note that $v \in \Omega_{2}$ because $\operatorname{tr}(v)=\left(\operatorname{tr}(z)^{2}-2 \operatorname{det}(z)\right) / 2 t\left(\operatorname{det}(z)^{2}\right)>0$ and $\operatorname{det}(v)=1 / 4 t^{2}(\operatorname{det}(z))^{2}>0$. Also $\operatorname{det} B=\operatorname{det}(v)^{d}=2^{-2 d} t^{-2 d} \operatorname{det}(z)^{-2 d}$. But

$$
\int_{A_{1 / 2}} e^{-(B \cdot \xi, \xi)} d \xi=\pi^{d}(\operatorname{det} B)^{-1 / 2},
$$

so

$$
I=(2 \pi)^{d} \operatorname{det}(z)^{d} \int_{0}^{+\infty} e^{-1 / t} t^{\nu-1} d t=(2 \pi)^{d} \Gamma(-\nu) \operatorname{det}(z)^{d} .
$$

Now

$$
\begin{aligned}
K_{\nu}^{[3]}\left(x_{1} c_{1}+x_{2} c_{2}\right) & =(2 \pi)^{d} \Gamma(-\nu) \int_{\Omega_{2}} e^{-\operatorname{tr}(z) / \operatorname{det}(z)} \exp -(x, z) \operatorname{det}(z)^{\nu-1} d z \\
& =(2 \pi)^{d} \Gamma(-\nu) \int_{\Omega_{2}} e^{-\operatorname{tr}\left(z^{-1}\right)} \exp -(x, z) \operatorname{det}(z)^{\nu+d / 2-d / 2-1} d z \\
& =(2 \pi)^{d} \Gamma(-\nu) K_{\nu+d / 2}^{[2]}\left(x_{1} c_{1}+x_{2} c_{2}\right) .
\end{aligned}
$$

Theorem 4.1. It holds that

$$
K_{\nu}^{[3]}\left(t_{1}, t_{2}, t_{3}\right)=\sum_{j=1}^{4} a_{\nu}^{j} J_{\nu}^{[3, j]}\left(-t_{1}, t_{2},-t_{3}\right)+b_{\nu}^{j} t_{3}^{-\nu} J_{-\nu}^{[3, j]}\left(-t_{1}, t_{2},-t_{3}\right)
$$

with

$$
\begin{gathered}
a_{\nu}^{1}=b_{-\nu}^{1}=(2 \pi)^{3 d / 2} \Gamma(-v) \Gamma\left(-v-\frac{d}{2}\right) \Gamma(-v-d), \\
a_{\nu}^{2}=b_{-\nu}^{2}=(2 \pi)^{3 d / 2} \Gamma(-v) \Gamma\left(-v-\frac{d}{2}\right) \Gamma(\nu+d), \\
a_{\nu}^{3}=b_{-\nu}^{3}=(2 \pi)^{3 d / 2} \Gamma(-v) \Gamma\left(\nu+\frac{d}{2}\right) \Gamma(\nu), \\
a_{\nu}^{4}=b_{-\nu}^{4}=(2 \pi)^{3 d / 2} \Gamma(-v) \Gamma\left(\nu+\frac{d}{2}\right) \Gamma(-v) .
\end{gathered}
$$

Proof. We suppose first that $\operatorname{Re}(\nu)<-d$. So the left-hand side of the formula (4.3) is nothing but $(2 \pi)^{d} \Gamma(-\nu) K_{v+d / 2}^{[2]}\left(x_{1} c_{1}+x_{2} c_{2}\right)$ according to (4.10). We use then the expansion established in the case of rank-2 Jordan algebra (see [3]) and the reduction formulas (2.8) to (2.11) for the right-hand side of (4.3).

\section{Conclusion}

In light of the above calculations, we think that the complete answer to Herz's conjecture (higher ranks) can be fulfilled in two steps. First a recurrence formula between $K$ Bessel functions of rank $r$ and $r+1$ like formula (4.3). This is quite easy because the lemmas above can be written in higher ranks using some Jordan algebra indentities. But what seems to be very difficult is the second step: the complete and explicit resolution 


\section{$2870 K$-Bessel functions associated to a 3-rank Jordan algebra}

of the Bessel-Muirhead system. Here the recurrence does not work because the series method yields a very complicated recurrence system on the coefficients.

\section{Acknowledgment}

The author is grateful to the referees for their relevant and helpful remarks.

\section{References}

[1] T. E. Bengtson, Bessel functions on $P_{n}$, Pacific J. Math. 108 (1983), no. 1, 19-29.

[2] H. Dib, Fonctions de Bessel sur une algèbre de Jordan [Bessel functions on a Jordan algebra], J. Math. Pures Appl. (9) 69 (1990), no. 4, 403-448 (French).

[3] $\longrightarrow$ K-Bessel functions in two variables, Int. J. Math. Math. Sci. 2003 (2003), no. 14, 909916.

[4] J. Faraut and A. Korányi, Analysis on Symmetric Cones, Oxford Mathematical Monographs, The Clarendon Press, Oxford University Press, New York, 1994.

[5] J. Faraut and G. Travaglini, Bessel functions associated with representations of formally real Jordan algebras, J. Funct. Anal. 71 (1987), no. 1, 123-141.

[6] C. S. Herz, Bessel functions of matrix argument, Ann. of Math. (2) 61 (1955), no. 3, 474-523.

[7] A. T. James, A generating function for averages over the orthogonal group, Proc. Roy. Soc. London Ser. A 229 (1955), 367-375.

[8] A. Korányi, Transformation properties of the generalized Muirhead operators, Colloq. Math. 60/61 (1990), no. 2, 665-669.

[9] N. H. Mahmoud, Bessel systems for Jordan algebras of rank 2 and 3, J. Math. Anal. Appl. 234 (1999), no. 2, 372-390.

[10] R. J. Muirhead, Systems of partial differential equations for hypergeometric functions of matrix argument, Ann. of Math. Statistics 41 (1970), no. 3, 991-1001.

[11] Aspects of Multivariate Statistical Theory, Wiley Series in Probability and Mathematical Statistics, John Wiley \& Sons, New York, 1982.

Hacen Dib: Department of Mathematics, Faculty of Sciences, University of Tlemcen, BP 119, Tlemcen 13000, Algeria

E-mail address: h_dib@mail.univ-tlemcen.dz 


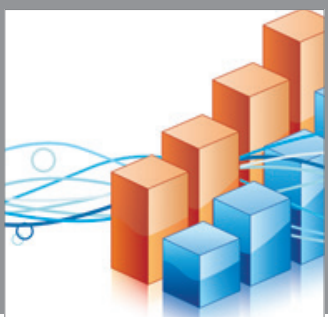

Advances in

Operations Research

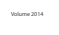

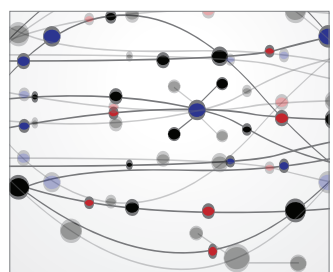

\section{The Scientific} World Journal
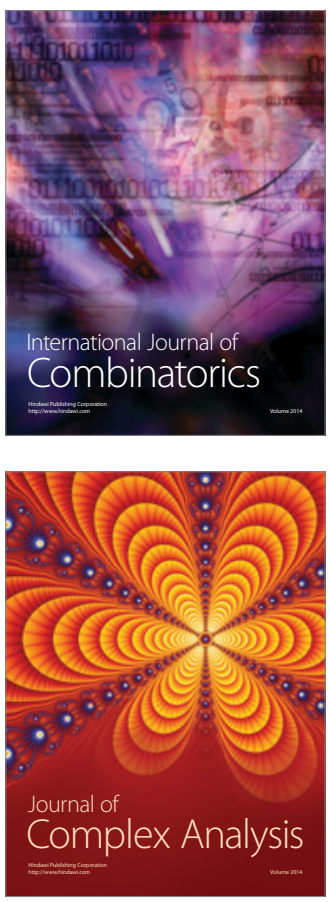

International Journal of

Mathematics and

Mathematical

Sciences
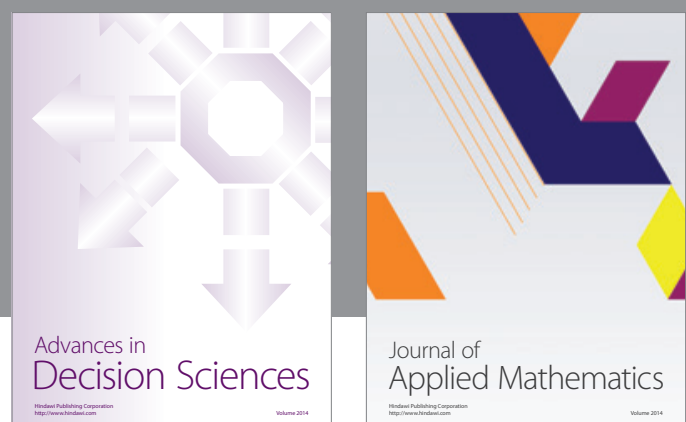

Journal of

Applied Mathematics
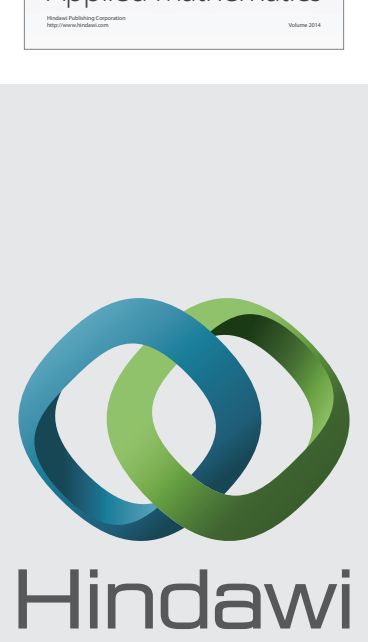

Submit your manuscripts at http://www.hindawi.com
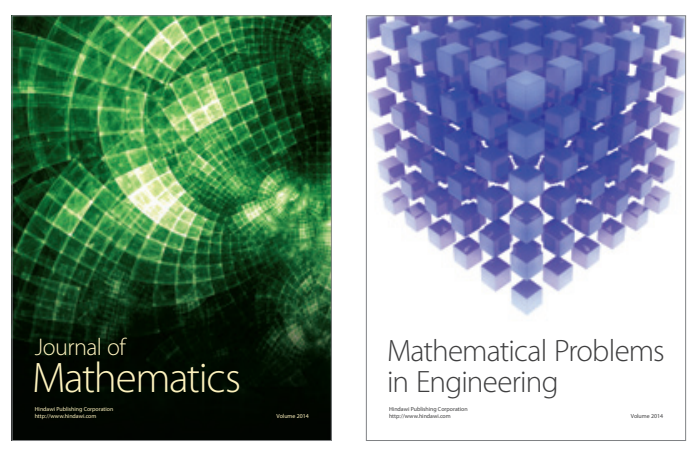

Mathematical Problems in Engineering
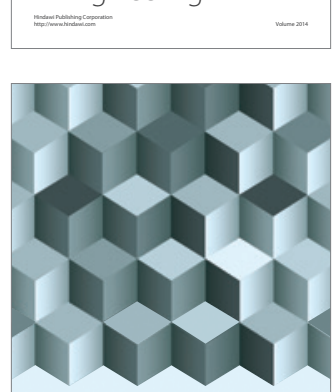

Journal of

Function Spaces
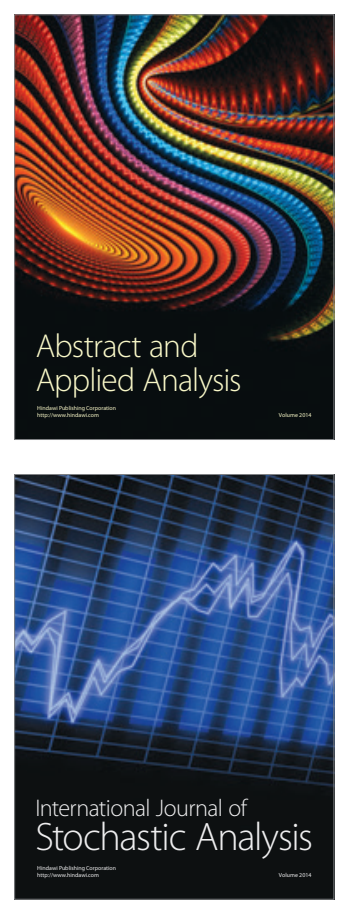

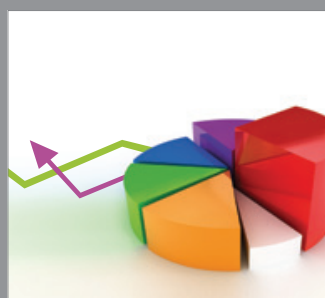

ournal of

Probability and Statistics

Promensencen
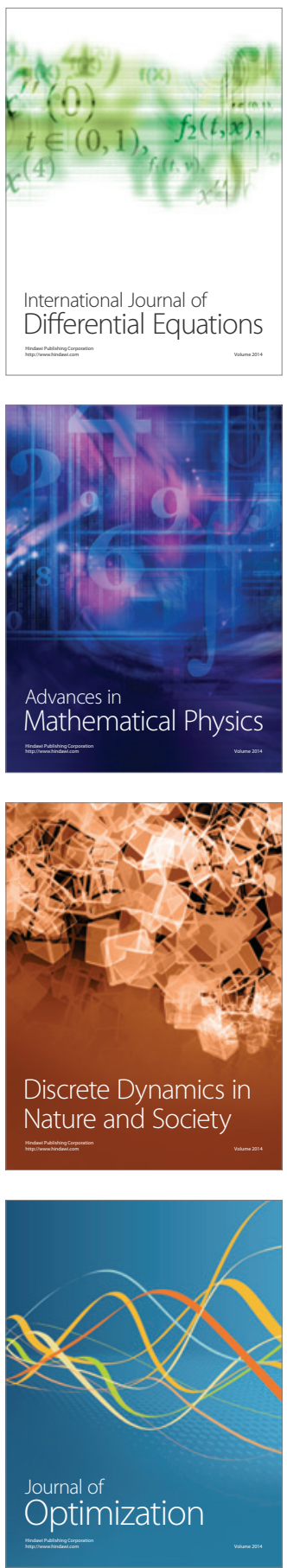